\title{
Optimizing Checkpoint Data Placement with Guaranteed Burst Buffer Endurance in Large-Scale Hierarchical Storage Systems
}

\author{
Lipeng Wan ${ }^{\mathrm{a}}$, Qing Cao ${ }^{\mathrm{a}}$, Feiyi Wang ${ }^{\mathrm{b}}$, Sarp Oral ${ }^{\mathrm{b}}$ \\ ${ }^{a}$ University of Tennessee, Knoxville \\ ${ }^{b}$ Oak Ridge National Laboratory
}

\begin{abstract}
Non-volatile devices, such as SSDs, will be an integral part of the deepening storage hierarchy on large-scale HPC systems. These devices can be on the compute nodes as part of a distributed burst buffer service or they can be external. Wherever they are located in the hierarchy, one critical design issue is the SSD endurance under the write-heavy workloads, such as the checkpoint I/O for scientific applications. For these environments, it is widely assumed that checkpoint operations can occur once every 60 minutes and for each checkpoint step as much as half of the system memory can be written out. Unfortunately, for large-scale HPC applications, the burst buffer SSDs can be worn out much more quickly given the extensive amount of data written at every checkpoint step. One possible solution is to control the amount of data written by reducing the checkpoint frequency. However, a direct effect caused by reduced checkpoint frequency is increased vulnerability window of system failures and therefore potentially wasted computation time, especially for large-scale compute jobs.

In this paper, we propose a new checkpoint placement optimization model which collaboratively utilizes both the burst buffer and the parallel file system to store the checkpoints, with design goals of maximizing computation efficiency while guaranteeing the SSD endurance requirements. Moreover, we present
\end{abstract}

Email addresses: lwan1@vols.utk.edu (Lipeng Wan), cao@utk.edu (Qing Cao), fwang2@ornl.gov (Feiyi Wang), oralhs@ornl.gov (Sarp Oral)

Preprint submitted to Journal of Parallel and Distributed Computing

August 25, 2016

(C) 2016. This manuscript version is made available under the Elsevier user license http://www.elsevier.com/open-access/userlicense/1.0/ 
an adaptive algorithm which can dynamically adjust the checkpoint placement based on the system's dynamic runtime characteristics and continuously optimize the burst buffer utilization. The evaluation results show that by using our adaptive checkpoint placement algorithm we can guarantee the burst buffer endurance with at most $5 \%$ performance degradation per application and less than $3 \%$ for the entire system.

Keywords: Fault Tolerance, Checkpoint, Hierarchical Storage Systems, Burst Buffer, Solid-State Drive

\section{Introduction}

Large-scale high performance computing (HPC) systems usually support running tens of scientific simulations on hundreds of thousands of compute nodes simultaneously. Due to the scale of both hardware and software components involved, failures are common and a fact of life in large-scale HPC systems' daily operation. Most scalable scientific applications cope with potential failures using some form of defensive programming technique - by periodically exporting their execution state and intermediary results as a "checkpoint" to a persistent storage. In the event of failures, they will be able to continue the execution (restart) without repeating previous computation.

Checkpoints generated by scientific applications are written to the parallel file systems (PFS) which are usually built using traditional storage servers and spinning disk drivers for balanced cost, performance, and capacity. Parallel file systems provide an efficient data access mechanism between various computation resources over high-performance storage area networks. However, given the frequency of the checkpoints and the amount of data written at each checkpoint step, the total checkpoint size written in an applications runtime can be daunting. Trying to absorb such large-scale checkpoint I/O with traditional parallel file systems can be cost-prohibitive. On the other hand, studies have shown that PFS has been underutilized in the sense that it operates in much lower bandwidth spectrum most of the time which is nowhere near the peak [1]. In 
order to resolve the dichotomy, the concept of "burst buffer" was recently proposed and has been designed and prototyped in some large-scale HPC systems $[1,2,3,4,5]$. The basic idea behind the "burst buffer" is that we can build an intermediate hardware and I/O middleware layer between compute nodes and parallel file systems to better handle I/O workloads from scientific applications by utilizing flash or SSD. The checkpoint data from scientific applications will be temporarily written into the burst buffer layer first and then drained to the underlying parallel file systems asynchronously. Since SSDs can provide much higher read and write bandwidth than hard disk drives, with the help of burst buffer layer, the I/O performance of scientific applications will be improved significantly, which also means the checkpointing can be done faster and more CPU time can be saved for computation.

Ideally, the burst buffer was designed to absorb all I/O workloads generated by large-scale applications running on supercomputers. However, in reality we may have to limit the amount of data written to the burst buffer if the endurance requirements on SSD devices are to be considered. Specifically, each block in a SSD must be erased before being rewritten and only a finite number of erasures are possible before the bit error of SSD becomes unacceptably high. Moreover, random writes could generate more erasure operations compared to sequential writes and lead to the increase of the so called write amplification [6]. In this paper, we focus our study on how general HPC I/O workloads, where a normal write amplification factor is often assumed, affect the lifetime of a typical SSD device. However, in some scenarios, different models of SSD devices might have different endurance even under the same I/O workload, or the inherent random access pattern of the $\mathrm{I} / \mathrm{O}$ workloads might make the endurance issue more severe than the gernal case studied here. Those special scenarios are out of this paper's scope, but will be studied in our future work. As an example, let us assume designing a burst buffer layer for a hypothetical large-scale HPC platform with tens of thousands of compute clients. If the building blocks are typical 256GB SSDs and if we are targeting a relatively moderate sized burst buffer layer (e.g. 5PB aggregate capacity), then the total number of SSDs 
required is about 20,000. According to the datasheet [7], the newest Samsung 850 Pro SSD (256GB) has a warranty for maximum 150TB write. If the burst buffer is designed to serve 5 years, the maximum amount of data that can be written to the entire burst buffer per day is 1,600TB. We further assume that the write amplification factor is around $1.3[6]$, then the actual allowed write is about 1,200TB per day. On the other hand, some common largescale scientific applications often produce huge checkpoint data. For example, if half of the compute nodes in ORNL's Titan [8] supercomputer are used to run the CHIMERA application [9], the size of each checkpoint generated at each checkpoint step is almost 160TB [10]. This is a common case when scientific applications are run at large-scale, since at each checkpoint step half of the data residing in the system memory can be written to the storage as a checkpoint. If several such scientific applications run simultaneously, the total size of the write workloads per day will be much larger than the SSD endurance requirements. Therefore, without constraints, the intensive write workloads produced by largescale long-running scientific applications through checkpointing could degrade the endurance of SSD devices and the reliability of the burst buffer significantly.

Several techniques and approaches $[11,12,13]$ have been proposed to optimize the endurance of SSD devices under different I/O workloads, particularly the kinds of workloads produced by personal computers, web servers, database systems, etc. However, none of them tackles on SSD endurance issues in HPC environment, because the HPC I/O workloads usually consist of extremely intensive write operations which can quickly wear out the SSD devices even when cutting-edge endurance optimization techniques are used. In fact, the HPC community does not have a full understanding of how to effectively maintain sustainable cost-to-performance and cost-to-capacity ratios for SSD devices under such write-heavy I/O workloads. One possible solution might be replacing the worn-out SSDs often to maintain a given capacity level, however, this solution is not feasible or cost-effective. The system-exclusive burst buffer can be built either by using node-local SSDs (i.e, an SSD device on every compute node) or can be shared (i.e, a set of pool of SSDs serving all compute nodes 
in a given HPC system). In the node-local case, the number of SSDs required grows linearly with the number of compute nodes. For the shared case, the required number of SSDs will grow linearly with the total memory size to absorb and flush the output data burst. In either case, we end up with thousands or tens of thousands of SSDs for a large-scale HPC system. To maintain the wear-out levels of this number of SSDs in a large-scale HPC facility will require extensive resources (i.e., man power to monitor and physically replace the worn-out devices on regular basis). Also, this approach will incur additional costs of the replaced devices. As an example, a modest size SSD can easily cost a few hundred U.S. dollars today and the replacing just the half of a 5,000 SSD population will amount to a few million U.S. dollars. Moreover, this approach requires compute node downtimes and interruptions to replace the worn-out devices from otherwise a "healthy" node (in terms of remaining components, such as CPU and memory), which is also an additional but hidden cost for the total cost of ownership (TCO) of a large-scale HPC system. For all these reasons combined, solely relying on physically replacing worn-out SSDs to maintain a set of required capacity and endurance targets is not cost-effective or practical.

Besides frequently replacing worn-out SSD devices, another possible solution would be reducing the amount of data written to the burst buffer. In [14], the authors proposed a checkpoint interval optimization model for large-scale scientific applications which takes the constraint of burst buffer capacity into consideration. In such model, SSD-based burst buffers of supercomputers are used to absorb all checkpoint data of the scientific applications. Therefore, in order to satisfy the capacity constraint, the model intends to reduce the checkpointing frequency of some write-heavy jobs so that the amount of data written to the burst buffer can be reduced. However, a direct effect caused by such reduction in checkpointing frequency is that the potential wasted computation time due to system failures also increases significantly, especially for large computation jobs.

In this paper, we propose a new checkpoint placement optimization model which collaboratively utilizes both the burst buffer and parallel file system in a 
large-scale storage system to store the checkpoint data generated by scientific applications. Specifically, our model guarantees the endurance requirements of the SSD-based burst buffer layers without sacrificing too much of the computational efficiency. Moreover, in order to make the model feasible to real HPC systems, we also design an adaptive algorithm which can dynamically adjust the checkpoint placement based on the changing runtime characteristics of the HPC system and continuously optimize the usage of the burst buffer. The evaluation results demonstrate the effectiveness of our checkpoint placement model and adaptive checkpoint placement algorithm. Particularly, using our adaptive checkpoint placement algorithm can guarantee the endurance of the burst buffer with at most $5 \%$ performance degradation of each job. Even better is that the degradation of the overall system computation efficiency is less than $3 \%$ if this adaptive algorithm is used. The remainder of this paper is organized as follows. In section 2 we provide a survey on existing studies that concentrate on improving the endurance of SSD devices. We also introduce models and algorithms that are commonly used to optimize the checkpoint/restart operations for large-scale scientific applications in section 3. We propose our checkpoint placement optimization model in section 4 and section 5 presents our adaptive optimization algorithm for the checkpoint placement. We evaluate our model and algorithm in section 6 and conclude our observation in section 7 .

\section{Related Work}

The idea of utilizing SSD devices to build a burst buffer layer between HPC systems and parallel file systems to temporarily absorb checkpoint I/O workloads has been researched over the last few years[1, 2, 3]. In fact, several recent high performance computing system deployments have already included burst buffer layers $[4,5]$. Existing studies in this area mainly focus on how to maximize the I/O performance of the checkpointing operations through the burst buffer $[15,16,17]$. For example, Scalable Checkpoint/Restart library (SCR) [15] provides an interface that allows scientific applications to periodically do check- 
pointing to SSDs, and asynchronously flush these checkpoints from SSDs to the underlying parallel file systems without interfering with applications' computation phase. In [16], a new design of the burst buffer system named BurstMem is proposed which implements functionalities similar to SCR but provides better I/O performance through efficient storage and communication management strategies. [17] presents a trace-driven performance evaluation of scientific I/O workloads on SSDs, which shows the concurrent I/O might significantly affect the SSD performance. However, none of these studies considered the endurance issue of the burst buffer under scientific I/O workloads, though such issue has emerged and will become extremely critical in next-generation HPC computing and storage systems.

For a single SSD device under common I/O workload, its endurance and reliability have been extensively studied. The existing body of work in this area can be classified into three categories. The first category focuses on improving the internal design of SSD devices [18, 19, 20], including designing better flash translation layers (FTL), more efficient wear-leveling and garbage collection algorithms. The second category mainly concentrates on OS-level optimizations [21, 22], including utilizing TRIM commands from OS, designing filesystemaware garbage collection algorithms. Finally, the third category reshapes the $\mathrm{I} / \mathrm{O}$ workloads $[23,24,11,25]$, including reducing the write workloads and the randomness of the access pattern, so that the write-amplification can be reduced. All of these techniques are effective if the amount of data written to SSDs is within some boundaries. However, for I/O workloads generated by largescale long-running scientific applications, these techniques might not be efficient, given the checkpoint frequency and amount of data written at each checkpoint step, consuming the allowed program-erase $(\mathrm{P} / \mathrm{E})$ cycles of underlying burst buffer SSDs and quickly wearing them out.

In order to better utilize SSD devices under scientific I/O workloads, [14] presents a checkpoint interval optimization model for large-scale scientific applications. This model is essentially same as those developed by [26, 27, 28] whose objective is to maximize the computational efficiency of HPC systems, but it 
also takes the constraint of burst buffer capacity into consideration. By using this model, optimal SSD capacity allocation among all scientific applications can be determined. Since the SSD-based burst buffers of the HPC systems are assumed to absorb all checkpoint data of the scientific applications, this model intends to reduce the checkpoint frequency of some write-heavy jobs if a rigorous constraint of burst buffer capacity is given. However, such reduction in checkpoint frequency also significantly increases the potential wasted computation time caused by system failures, especially for large computation jobs.

Additionally, most of the checkpoint interval optimization models, including those mentioned above, are static, meaning the checkpoint interval is determined through one-time calculation when the scientific job is launched and kept unchanged during the job's execution. Due to the dynamic runtime characteristics of the HPC system, static checkpoint optimization usually cannot achieve the best system efficiency. Therefore, adaptive checkpoint interval optimization has also been studied in $[29,30,31]$. However, few of them takes the endurance issue of the SSD devices into account, which makes their models or algorithms not applicable to the next-generation HPC systems where SSD devices are indispensable components.

In summary, little work has been done to address critical design issue on system-level endurance and reliability involving SSD devices, especially when used in the burst buffer layer under the write-heavy scientific I/O workloads in HPC environment, which is the focus and thesis of this paper.

\section{Background}

Before we discuss our checkpoint placement optimization model with guaranteed burst buffer endurance, we first briefly introduce existing studies on determining the optimal checkpoint interval and how to adjust the checkpoint intervals when different constraints are taken into consideration. The notations of all the symbols used in the following sections are listed in TABLE 1. 


\begin{tabular}{|c|c|}
\hline$M$ & $\begin{array}{l}\text { Total number of scientific application jobs } \\
\text { running on the HPC system }\end{array}$ \\
\hline$N$ & Total number of compute nodes in the HPC system \\
\hline$\lambda$ & Failure rate per compute node in the HPC system \\
\hline$l_{\max }$ & Daily write limits of the burst buffer \\
\hline$n_{i}$ & Number of compute nodes assigned to execute the $i$-th job \\
\hline$T_{c m p t, i}$ & Total computation time required to finish the $i$-th job \\
\hline$\Delta t_{c m p t, i}$ & Checkpoint interval of the $i$-th job \\
\hline$S_{i}$ & Data size of one checkpoint from the $i$-th job \\
\hline$\alpha_{i}$ & $\begin{array}{l}\text { Percentage of checkpoints from the } i \text {-th job } \\
\text { that should be written to the burst buffer }\end{array}$ \\
\hline$t h r p t_{i}^{w r, b b}$ & $\begin{array}{l}\text { Throughput when the } i \text {-th job writes checkpoints to } \\
\text { the burst buffer }\end{array}$ \\
\hline thrpt $t_{i}^{w r, p f s}$ & $\begin{array}{l}\text { Throughput when the } i \text {-th job writes checkpoints to } \\
\text { the parallel file systems }\end{array}$ \\
\hline$\Delta t_{c k p t, i}^{w r, b b}$ & $\begin{array}{l}\text { Time required to write one checkpoint of the } \\
i \text {-th job to the burst buffer }\end{array}$ \\
\hline$\Delta t_{c k p t, i}^{w r, p f s}$ & $\begin{array}{l}\text { Time required to write one checkpoint of the } \\
i \text {-th job to the parallel file systems }\end{array}$ \\
\hline
\end{tabular}

Table 1: Notations of symbols

\subsection{Optimal Checkpoint Interval}

Most existing studies on finding optimal checkpoint interval model the execution of each large-scale scientific application as a sequence of activities alternating between the computation and checkpoint phases. Here we use $\Delta t_{c m p t, i}$ to denote the computation time period between two consecutive checkpoint activities of the $i$-th job, which is also referred to as the checkpoint interval of the $i$-th job. We use $\Delta t_{c k p t, i}^{w r}$ to denote the time spent on writing one checkpoint of the $i$-th job to the storage system.

Both hardware and software failures of compute nodes could interrupt the computation or checkpoint activities and trigger a restart (reading the latest checkpoint from the storage system to re-launch the job from the last correct state). As shown in Figure 1, if we assume $\epsilon$ percent of computation and checkpoint activities are wasted on average due to failures, we can 


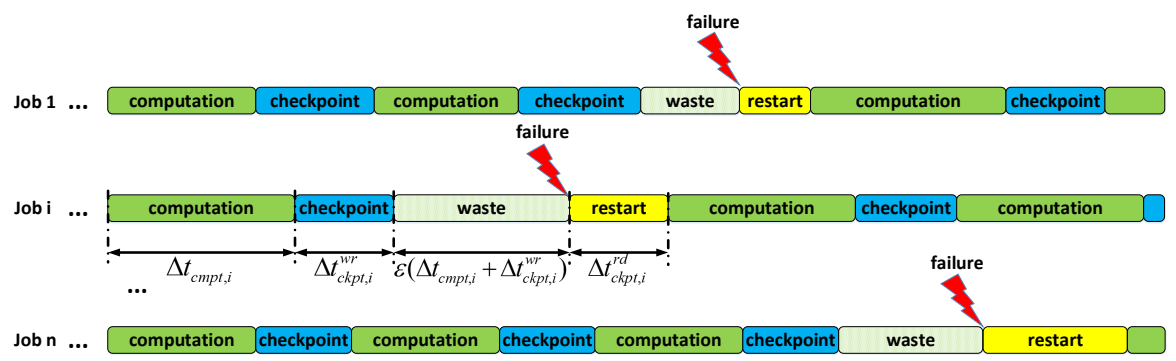

Figure 1: Checkpoint/restart for scientific applications running on HPC systems

then calculate the overhead caused by one failure of $i$-th job as $\Delta t_{\text {overhead }, i}=$ $\epsilon\left(\Delta t_{c m p t, i}+\Delta t_{c k p t, i}^{w r}\right)+\Delta t_{c k p t, i}^{r d}$, where $\Delta t_{c k p t, i}^{r d}$ is the time required to read one checkpoint of the $i$-th job from the storage system (usually $\Delta t_{c k p t, i}^{r d}$ can be ignored since $\left.\Delta t_{c k p t, i}^{r d} \ll \Delta t_{c m p t, i}+\Delta t_{c k p t, i}^{w r}\right)$.

Most existing work assume the arrival of system failures follows a Poisson distribution and on average half of the work after last checkpoint is wasted if a failure occur $(\epsilon=0.5)[26,27,28,14]$. If the failure rate per compute node in the HPC system is $\lambda$ and the number of compute nodes occupied by the $i$-th job is $n_{i}$, the total execution time of the $i$-th job can be denoted as:

$$
T_{t o t a l, i}=T_{c m p t, i}\left(1+\frac{\Delta t_{c k p t, i}^{w r}}{\Delta t_{c m p t, i}}+\frac{1}{2} \lambda n_{i} \Delta t_{c m p t, i}\right)
$$

where $T_{c m p t, i}$ is the total computation time required to finish the $i$-th job, $\frac{\Delta t_{c k p t, i}^{w r}}{\Delta t_{c m p t, i}} T_{c m p t, i}$ is the total time spent on checkpointing, and $\frac{1}{2} \lambda n_{i} \Delta t_{c m p t, i} T_{c m p t, i}$ is the total overhead caused by the failures. According to [26, 27, 28], $T_{\text {total, } i}$ can be minimized when the following value is used as the checkpoint interval:

$$
\Delta t_{c m p t, i}=\sqrt{\frac{2 \Delta t_{c k p t, i}^{w r}}{\lambda n_{i}}}
$$

3.2. Identifying Checkpoint Intervals by Exploiting the Temporal Locality of Failures

In [10], the failure datasets collected from several HPC systems were analyzed. The results showed that the time between failures in HPC systems follow 
a Weibull distribution and the failure rate decreases over time since the last failure (and until the next failure). Therefore, [10] proposes an adaptive checkpoint model called "lazy checkpointing", which increases the checkpoint interval until the next failure. The incrementally increasing checkpoint interval is given by the following formula:

$$
\Delta t_{c m p t, i}^{l a z y}=\Delta t_{c m p t, i}\left(\frac{t}{\Delta t_{c m p t, i}}\right)^{1-\beta},
$$

where $\beta$ is the shape parameter of the Weibull distribution, $t$ is the present time and $\Delta t_{c m p t, i}$ is the optimal checkpoint interval derived using formula (2). Once a failure happens, the checkpoint interval is reset to $\Delta t_{c m p t, i}$.

The basic idea behind the above formula is to increase the checkpoint interval over time so that it has the same slope as the curve of the failure rate. Since this "lazy checkpointing" model intends to skip some checkpoints, it can mitigate the $\mathrm{I} / \mathrm{O}$ overhead caused by checkpointing and improve the efficiency of the HPC system compared to the static optimal checkpoint interval represented by 2.

\subsection{Identifying Checkpoint Intervals for a Fixed Burst Buffer Capacity}

For absorbing the output of large-scale scientific applications with high checkpoint frequencies and large data sizes in each step, a burst buffer may require tens of thousands of SSD devices. Since SSDs are more expensive than traditional spinning disk drives of the same capacity, system designers often try to limit the number of SSDs. [14] follows a similar logic and tries to determine a new optimal checkpoint interval under a fixed burst buffer capacity. Their model is:

$$
\begin{aligned}
\underset{\Delta t_{c m p t, i}}{\arg \min } & \sum_{i=1}^{M} T_{\text {total }, i} \\
\text { s.t. } & \sum_{i=1}^{M} \frac{T_{c m p t, i}}{\Delta t_{c m p t, i}} S_{i} \leq C_{\text {max }},
\end{aligned}
$$

where $C_{\max }$ is the total capacity of SSDs provisioned for the burst buffer to store the checkpoint data. 
There are two disadvantages in this model. First, the objective function just simply sums up the total execution time of each job, which cannot accurately represent the total consumed computation resources. Instead, the execution time of each job should be weighted by the number of compute nodes each job occupies. Second, the above model intends to extend the checkpoint interval of each job, especially those with larger checkpoint data sizes or longer total computation times, to limit the amount of data written to the burst buffer, which significantly increases the potential wasted computation time caused by system failures.

In this work, we argue that the endurance is as critical as capacity in designing SSD-based burst buffers.

\section{Checkpoint Placement Optimization with Guaranteed Burst Buffer Endurance}

All existing studies assume that the burst buffer is used to absorb all checkpoint data from the HPC systems and only some of the checkpoints (e.g. every $n$th checkpoint) are flushed from the burst buffer to the underlying parallel file systems. In that case, if multiple jobs that all produce large amounts of checkpoint data execute concurrently, then it becomes very challenging to maintain the burst buffer endurance requirements without negatively affecting the computational efficiency. Therefore, in order to optimize the computational efficiency of large-scale scientific applications while guaranteeing the lifetime of the SSDbased burst buffer, we propose a new optimization model which collaboratively leverages both the burst buffer and parallel file systems to store checkpoint data. A major difference from existing approaches is that our proposed model can keep the original optimal checkpoint interval (to the best it can) and also reduces the potential wasted computation time caused by system failures without exceeding the write limit of the burst buffer.

We denote the time consumed by writing one checkpoint of the $i$-th job to

the burst buffer as $\Delta t_{c k p t, i}^{w r, b b}$ and that to the parallel file systems as $\Delta t_{c k p t, i}^{w r, p f s}$. If 
the write throughput of the $i$-th job to the burst buffer and parallel file systems are given, we can calculate $\Delta t_{c k p t, i}^{w r, b b}$ and $\Delta t_{c k p t, i}^{w r, p s}$ as:

$$
\begin{aligned}
\Delta t_{c k p t, i}^{w r, b b} & =\frac{S_{i}}{t h r p t_{i}^{w r, b b}}, \\
\Delta t_{c k p t, i}^{w r, p f s} & =\frac{S_{i}}{\operatorname{thrpt}_{i}^{w r, p f s}}
\end{aligned}
$$

The challenge is to determine the optimal percentage of the checkpoints that should be written to the burst buffer per application. If we use $\alpha_{i}$ to denote the percentage of checkpoints from the $i$-th job which should be written to the burst buffer, then the average time spent on writing one checkpoint of the $i$-th job, $\Delta t_{c k p t, i}^{w r}$, can be calculated as:

$$
\Delta t_{c k p t, i}^{w r}=\alpha_{i} \Delta t_{c k p t, i}^{w r, b b}+\left(1-\alpha_{i}\right) \Delta t_{c k p t, i}^{w r, p f s}
$$

If we replace $\Delta t_{c m p t, i}$ with $\sqrt{\frac{2 \Delta t_{c k p t, i}^{w r}}{\lambda n_{i}}}$ in (1), we can obtain the total execution time of the $i$-th job as:

$$
\begin{aligned}
T_{\text {total }, i} & =T_{c m p t, i}\left(1+\frac{\Delta t_{c k p t, i}^{w r}}{\Delta t_{c m p t, i}}+\frac{1}{2} \lambda n_{i} \Delta t_{c m p t, i}\right) \\
& =T_{c m p t, i}\left(1+\frac{\Delta t_{c k p t, i}^{w r}}{\sqrt{\frac{2 \Delta t_{c k p t, i}^{w r}}{\lambda n_{i}}}}+\frac{1}{2} \lambda n_{i} \sqrt{\frac{2 \Delta t_{c k p t, i}^{w r}}{\lambda n_{i}}}\right) \\
& =T_{c m p t, i}\left(1+\sqrt{2 \lambda n_{i} \Delta t_{c k p t, i}^{w r}}\right) \\
& =T_{c m p t, i}\left\{1+\sqrt{2 \lambda n_{i}\left[\alpha_{i} \Delta t_{c k p t, i}^{w r, b b}+\left(1-\alpha_{i}\right) \Delta t_{c k p t, i}^{w r, p f s}\right]}\right\}
\end{aligned}
$$

We formulate the objective function of our optimization model as follows

$$
\underset{\alpha_{i}}{\arg \min } \sum_{i=1}^{M} n_{i} T_{c m p t, i}\left\{1+\sqrt{2 \lambda n_{i}\left[\alpha_{i} \Delta t_{c k p t, i}^{w r, b b}+\left(1-\alpha_{i}\right) \Delta t_{c k p t, i}^{w r, p f s}\right]}\right\},
$$

in which each job's total execution time is weighted by the number of compute nodes they occupies.

The total size of the checkpoint data written to the burst buffer can be calculated as

$$
\sum_{i=1}^{M} \frac{T_{c m p t, i}}{\sqrt{\frac{2\left[\alpha_{i} \Delta t_{c k p t, i}^{w r, b b}+\left(1-\alpha_{i}\right) \Delta t_{c k p t, i}^{w r, p f s}\right]}{\lambda n_{i}}}} \alpha_{i} S_{i}
$$


Since vendors provide the daily write limits of the SSD devices, we denote the daily write limit of the entire SSD-based burst buffer as $l_{\max }$. After we divide (11) by $T_{c m p t, i}$, we can obtain the average checkpoint data written per hour, which should not exceed the per-hour write limit of the burst buffer, as the constraint of our model:

$$
\sum_{i=1}^{M} \frac{\alpha_{i} S_{i}}{\sqrt{\frac{2\left[\alpha_{i} \Delta t_{c k p t, i}^{w r, b b}+\left(1-\alpha_{i}\right) \Delta t_{c k p t, i}^{w r, p f s}\right]}{\lambda n_{i}}}} \leq l_{\max } / 24
$$

Putting it all together, we establish the following optimization model to determine the optimal percentage of an application checkpoint that should be written to the burst buffer.

$$
\begin{array}{r}
\underset{\alpha_{i}}{\arg \min } \sum_{i=1}^{M} n_{i} T_{c m p t, i}\left\{1+\sqrt{2 \lambda n_{i}\left[\alpha_{i} \Delta t_{c k p t, i}^{w r, b b}+\left(1-\alpha_{i}\right) \Delta t_{c k p t, i}^{w r, p f s}\right]}\right\} \\
\text { s.t. } \sum_{i=1}^{M} \frac{\alpha_{i} S_{i}}{\sqrt{\frac{2\left[\alpha_{i} \Delta t_{c k p t, i}^{w r, b b}+\left(1-\alpha_{i}\right) \Delta t_{c k p t, i}^{w r, p f s}\right]}{\lambda n_{i}}}} \leq l_{\max } / 24
\end{array}
$$

Apparently, our optimization model is a nonlinear programming model. To make it more easier to resolve, we substitute $\sqrt{2 \lambda n_{i}\left[\alpha_{i} \Delta t_{c k p t, i}^{w r, b b}+\left(1-\alpha_{i}\right) \Delta t_{c k p t, i}^{w r, p f s}\right]}$ with $x_{i}$ in both the objective function and constraint to transform the model into the following form:

$$
\begin{aligned}
\underset{\alpha_{i}}{\arg \min } & \sum_{i=1}^{M} n_{i} T_{c m p t, i}\left(1+x_{i}\right) \\
\text { s.t. } & \sum_{i=1}^{M} \frac{\frac{1}{2} x_{i}-\frac{\lambda n_{i} t_{c k p t, i}^{w r, p f s}}{x_{i}}}{t_{c k p t, i}^{w r, b b}-t_{c k p t, i}^{w r, p f s}} S_{i} \leq l_{\max } / 24
\end{aligned}
$$

We can solve this constrained nonlinear programming problem by using the interior-point algorithm [32].

After we obtain the optimal value for $x_{i}$, thereby $\alpha_{i}$, according to formula (2), we can also calculate the new optimal checkpoint interval of the $i$-th job as

$$
\Delta t_{c m p t, i}=\sqrt{\frac{2\left(\alpha_{i} \Delta t_{c k p t, i}^{w r, b b}+\left(1-\alpha_{i}\right) \Delta t_{c k p t, i}^{w r, p f s}\right)}{\lambda n_{i}}}
$$


By using both the optimal $\alpha_{i}$ and $\Delta t_{c m p t, i}$ for all scientific applications, we can maximize the computation efficiency of an HPC system while guaranteeing the SSD endurance requirements for the burst buffer.

\section{Adaptive Checkpoint Placement for Optimal HPC System and Burst Buffer Usage}

Assuming runtime characteristics, such as failure rates, job size, and checkpoint size, of scientific applications are given, we can solve the above optimization model and determine checkpoint data placement, checkpoint interval and the percentage of checkpoint data that should be stored on the burst buffer on a per job basis. However, in practice, some of these runtime characteristics cannot be obtained before execution and others vary with time. Therefore, we would like to design an adaptive algorithm which can dynamically adjust the checkpoint placement based on those time-dependent characteristics and continuously optimize the usage of the burst buffer.

\subsection{Runtime Characteristics of HPC Systems and Scientific Applications}

First, let us categorize the runtime characteristics of HPC systems and scientific applications that are required for determining the optimal checkpoint placement and identify the ones varying during the application execution.

- Checkpoint size: Although different scientific applications write checkpoints of different sizes, for a specific scientific application the size of each checkpoint is usually constant.

- Job size: Job size means the number of compute nodes each computation job occupies. Job size not only determines the aggregate I/O write and read bandwidths, but also effects the failure rate of each running scientific application. The job size of each application is usually determined before the execution and does not change if the computation continues normally. However, when the job is restarting from a failure, the job size might be 
changed as it depends on how the job scheduler allocates the compute nodes to restart the job.

- Aggregate I/O bandwidth: The aggregate I/O bandwidth that each job can achieve when writing checkpoints to either the burst buffer or the parallel file system are determined by the job size. Jobs with larger sizes often have higher aggregate $\mathrm{I} / \mathrm{O}$ bandwidth. If the job size remains the same, the aggregate I/O bandwidth for each job will not change.

- Total computation time: Total computation time of each scientific application is the time required to finish all computation tasks, not including that spent on writing checkpoints or recovering from failures. It depends on the complexity of the job and the job size. If the job size does not change, this value for each scientific application will be constant.

- Per-node failure rate: Per-node failure rate of HPC systems is often estimated using historical failure logs. Though constant failure rates are often assumed in most of existing models, several studies have shown that the failure rates of compute nodes in large-scale HPC systems might vary with time $[33,10,34]$. For instance, [10] presents the analysis of failure data collected from multiple supercomputing facilities including Oak Ridge Leadership Computing Facility (OLCF) and the Los Alamos National Laboratory (LANL). The results indicate that there is a strong temporal locality between compute node failures in HPC systems.

\subsection{Effect of Dynamic Runtime Characteristics on Checkpoint Placement Op- timization}

From the above analysis, we realize that except the checkpoint size, all other runtime characteristics might change during the execution. Next, we need to study how the variation in these runtime characteristics effect the optimization results of the checkpoint placement. 


\subsubsection{When job size is changed}

The job size could be changed when the job is restarting from a failure. For example, on Titan supercomputer, when a job is terminated by a failure, the system might try to restart it by resubmitting it to the job scheduler. Then the job scheduler will re-launch the job on currently available compute nodes, which usually are not the nodes used by the job before the failure. If the available compute nodes are less than those used before the failure, the size of the relaunched job has to be downgraded.

In the optimization model given by formula (13), the size of the $i$-th job is denoted by $n_{i}$. If $n_{i}$ decreases, the aggregate failure rate of the $i$-th job, $\lambda n_{i}$, will decrease. Meanwhile, the decrease of $n_{i}$ also reduces the aggregate $\mathrm{I} / \mathrm{O}$ bandwidth the $i$-th job can achieve, thereby increases the average time spent on writing checkpoints to the storage, $\left[\alpha_{i} \Delta t_{c k p t, i}^{w r, b b}+\left(1-\alpha_{i}\right) \Delta t_{c k p t, i}^{w r, p f s}\right]$. Therefore, if $n_{i}$ decreases, according to the constraint of the optimization model, the checkpoints written to the burst buffer will decrease. In that case, if we do not write more percentage of checkpoints to the burst buffer, the burst buffer will be underutilized.

\subsubsection{When an existing job finishes or a new job joins}

If an existing job finishes, all the computing resources it occupies will be released. It does not need the burst buffer to store its checkpoints anymore. In that case, the checkpoint placement should be re-optimized and the new percentage of checkpoint data that should be stored on the burst buffer as well as the new checkpoint interval should be calculated for those remaining jobs. Basically, the remaining jobs will be allocated more burst buffer write permit since the total write workload to the burst buffer has decreased.

Similarly, when a new job joins, the optimization results on checkpoint placement should be calculated again and some burst buffer write permit should be allocated to the new job accordingly. 


\subsubsection{When failure rate is time-dependent}

Time-dependent failure rates have been observed among compute nodes in HPC systems of different sizes. For example, we analyze the failure logs collected from three different HPC clusters, LANL's system 8, 18, and OLCF's Titan. These three clusters are equiped with different number of compute nodes, and can represent small, medium and large scale HPC system respectively. Though Titan's failure logs are not publicly available, the failure logs of LANL's HPC systems are availalbe online and more details can be found in [35]. We use four different distributions to fit the time between failures (in hours) extracted from the failure logs. The fitting results are shown in TABLE 2, which lists the parameters of each distribution estimated by using Maximum Likelihood Estimation. In order to illustrate the fitness of these four different distributions, we present the Q-Q plot for each distribution in Figure 2. Specifically, each Q-Q plot compares the quantiles drawn from the failure data (y-axis) to theoretical quantiles calculated from a particular distribution using parameters listed in TABLE 2 (x-axis). If the quantiles of the failure data came from the same distribution, the points in the Q-Q plot will approximately lie on the line $y=$ $x$. As shown in Figure 2, for each of these three HPC systems, the Weibull distribution fits the failure data best.

\begin{tabular}{|c|c|c|c|c|}
\hline \multirow{2}{*}{ System } & \multicolumn{4}{|c|}{ Distribution } \\
\hline & Exponential & Weibull & Normal & Lognormal \\
\hline $\begin{array}{l}\text { LANL System } 8 \\
\text { (164 nodes) }\end{array}$ & rate $=0.0119$ & $\begin{array}{l}\text { shape }=0.7111, \\
\text { scale }=67.375\end{array}$ & $\begin{array}{c}\operatorname{mean}=84.079, \\
\mathrm{sd}=122.00\end{array}$ & $\begin{array}{c}\text { meanlog }=3.3992, \\
\text { sdlog }=1.7931\end{array}$ \\
\hline $\begin{array}{l}\text { LANL System } 18 \\
\text { (1024 nodes })\end{array}$ & rate $=0.1336$ & $\begin{array}{l}\text { shape }=0.8170, \\
\text { scale }=6.6293\end{array}$ & $\begin{array}{c}\text { mean }=7.4829, \\
\mathrm{sd}=12.806\end{array}$ & $\begin{array}{c}\text { meanlog }=1.2194, \\
\text { sdlog }=1.4505\end{array}$ \\
\hline $\begin{array}{l}\text { OLCF Titan } \\
(18,688 \text { nodes })\end{array}$ & rate $=0.1378$ & $\begin{array}{l}\text { shape }=0.6885 \\
\text { scale }=5.4527\end{array}$ & $\begin{array}{c}\text { mean }=7.2565 \\
\mathrm{sd}=12.731\end{array}$ & $\begin{array}{c}\text { meanlog }=0.9197 \\
\text { sdlog }=1.5817\end{array}$ \\
\hline
\end{tabular}

Table 2: Distributions fitted to the failure data

For each of these three HPC systems given in TABLE 2, the shape parameter of the Weibull distribution is less than 1 , indicating a decreasing failure rate. 

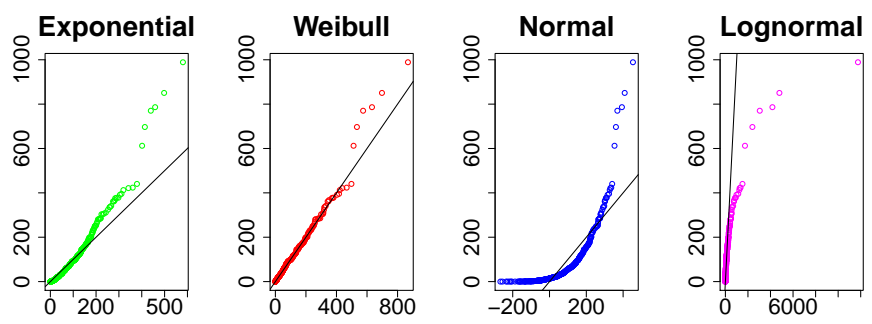

(a) LANL System 8 (164 nodes, small scale)
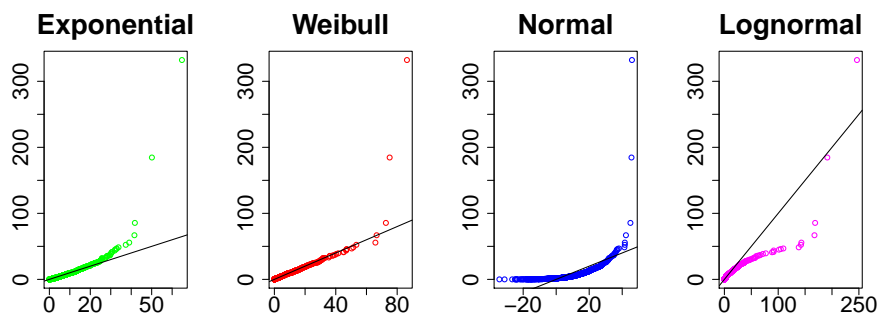

(b) LANL System 18 (1024 nodes, medium scale)
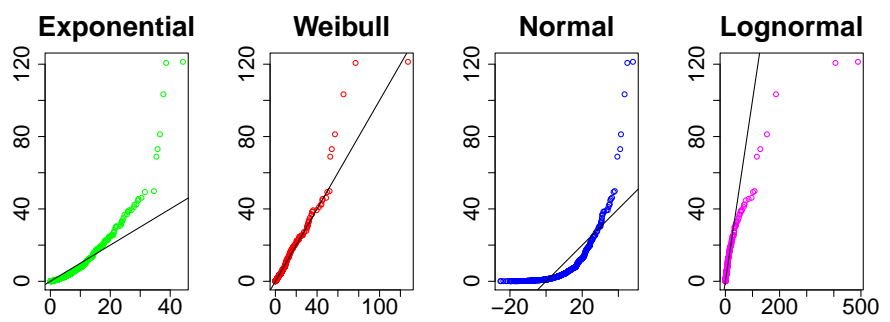

(c) OLCF Titan (18,688 nodes, large scale)

Figure 2: Q-Q plot for visualizing fitness of different distributions

This means the expected remaining time until the next failure increases with the time since the last failure has occurred, or in other words, the next failure is more likely to happen within a relatively short time period after the last failure. For example, as shown in Figure 3, if failure 2 is the next failure after the failure 1 , then it is more likely to occur very soon after failure 1 because of the decreasing failure rate.

Ideally, the most efficient way to utilize the burst buffer is limiting the write workloads to extend the SSDs' lifetime while maximizing the read workloads to 


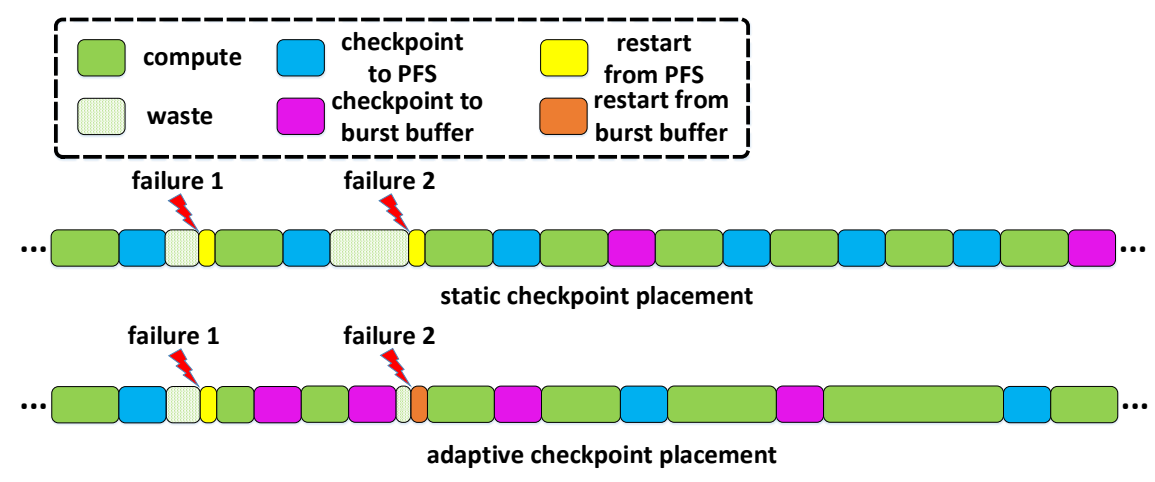

Figure 3: Static/adaptive checkpoint placement

reduce the job restarting time to leverage the high read throughput of the burst buffer. However, if the static checkpoint placement is used, as shown in Figure 3 , the checkpoints are written with constant frequency and a fixed percentage of them are stored on the burst buffer. In this case, the probability that the job uses a checkpoint stored on the burst buffer to recover from the failure is low since the failures are not uniformly distributed due to the decreasing failure rate. Therefore, in order to utilize the HPC system and the burst buffer more efficiently, the checkpoint placement algorithm must also be able to adapt to the time-dependent failure rate. For instance, as shown in Figure 3, the adaptive checkpoint placement should be able to adjust the percentage of checkpoints written to the burst buffer after failure 1, so that the likelihood of restarting the job using checkpoints from the burst buffer when failure 2 happens can be increased.

\subsection{Adaptive Checkpoint Placement Optimization Algorithm}

The design of our adaptive checkpoint placement algorithm is based on two assumptions. First, in HPC systems, it is possible to obtain all running jobs' runtime characteristics (such as the job size, the remaining computation time, failure rate, etc.), which will be fed to our optimization algorithm as the input parameters. Second, there is a way to apply the optimization results produced by our algorithm to adjusting the checkpoint interval of each scientific com- 
putation job. In practice, the first assumption is often valid as most of the commonly used workload manager softwares are able to collect jobs' runtime information during operation. The second assumption is also possible as some workload managers, such as SLURM [36], can utilize interfaces provided by checkpoint/restart libraries, such as BLCR [37], to configure the checkpoint interval of each job. Therefore, it is possible to integrate our algorithm into the workload managers to achieve adaptive checkpoint placement optimization.

In order to make the checkpoint placement adaptive to those changing runtime characteristics, the previous model designed for the static checkpoint placement optimization needs to be modified. First, given a fixed optimization period, $\Delta_{o p t}$, the objective function of the model, as shown bellow, is to minimize the overhead, including the time spent on writing, reading checkpoints and the wasted computation time due to failures, during such time period.

$$
\begin{aligned}
\underset{\alpha_{i}}{\arg \min } \sum_{i=1}^{M} & n_{i}\left\{\frac{\Delta_{o p t}}{\Delta t_{c m p t, i}}\left[\alpha_{i} \Delta t_{c k p t, i}^{w r, b b}+\left(1-\alpha_{i}\right) \Delta t_{c k p t, i}^{w r, p f s}\right]+\right. \\
& \left.\frac{n_{i}}{N} N_{f}\left[\alpha_{i} \Delta t_{c k p t, i}^{r d, b b}+\left(1-\alpha_{i}\right) \Delta t_{c k p t, i}^{r d, p f s}\right]+\frac{n_{i}}{N} N_{f} \frac{\Delta t_{c m p t, i}}{2}\right\},
\end{aligned}
$$

where $N_{f}$ is the expected number of node failures during $\Delta_{o p t}$. If we assume the current time is $t_{\text {now }}$ and the last failure happened at $t_{f a i l}, N_{f}$ can be calculated as $N_{f}=\int_{t_{\text {now }}-t_{\text {fail }}}^{t_{\text {now }}+\Delta t_{\text {fail }}} h(t) d t$, where $h(t)$ is the hazard rate function estimated by using the failure logs collected from the Titan supercomputer $\left(h(t)=\frac{\beta}{\eta}\left(\frac{t}{\eta}\right)^{\beta-1}\right.$, where $\beta$ is the shape parameter and $\eta$ is the scale parameter of the Weibull distribution). The above objective function achieves the minimum when $\Delta t_{c m p t, i}=\sqrt{\frac{\Delta_{o p t}\left[\alpha_{i} \Delta t_{c k p t, i}^{w r, b b}+\left(1-\alpha_{i}\right) \Delta t_{c k p t, i}^{w r, p f s}\right]}{n_{i} N_{f} /(2 N)}}$, and after we replace the $\Delta t_{c m p t, i}$ with this value, the objective function can be simplified as:

$$
\begin{gathered}
\underset{\alpha_{i}}{\arg \min } \sum_{i=1}^{M} n_{i}\left\{\sqrt{2 \frac{n_{i}}{N} N_{f} \Delta_{o p t}\left[\alpha_{i} \Delta t_{c k p t, i}^{w r, b b}+\left(1-\alpha_{i}\right) \Delta t_{c k p t, i}^{w r, p f s}\right]}\right. \\
\left.+\frac{n_{i}}{N} N_{f}\left[\alpha_{i} \Delta t_{c k p t, i}^{r d, b b}+\left(1-\alpha_{i}\right) \Delta t_{c k p t, i}^{r d, p f s}\right]\right\}
\end{gathered}
$$




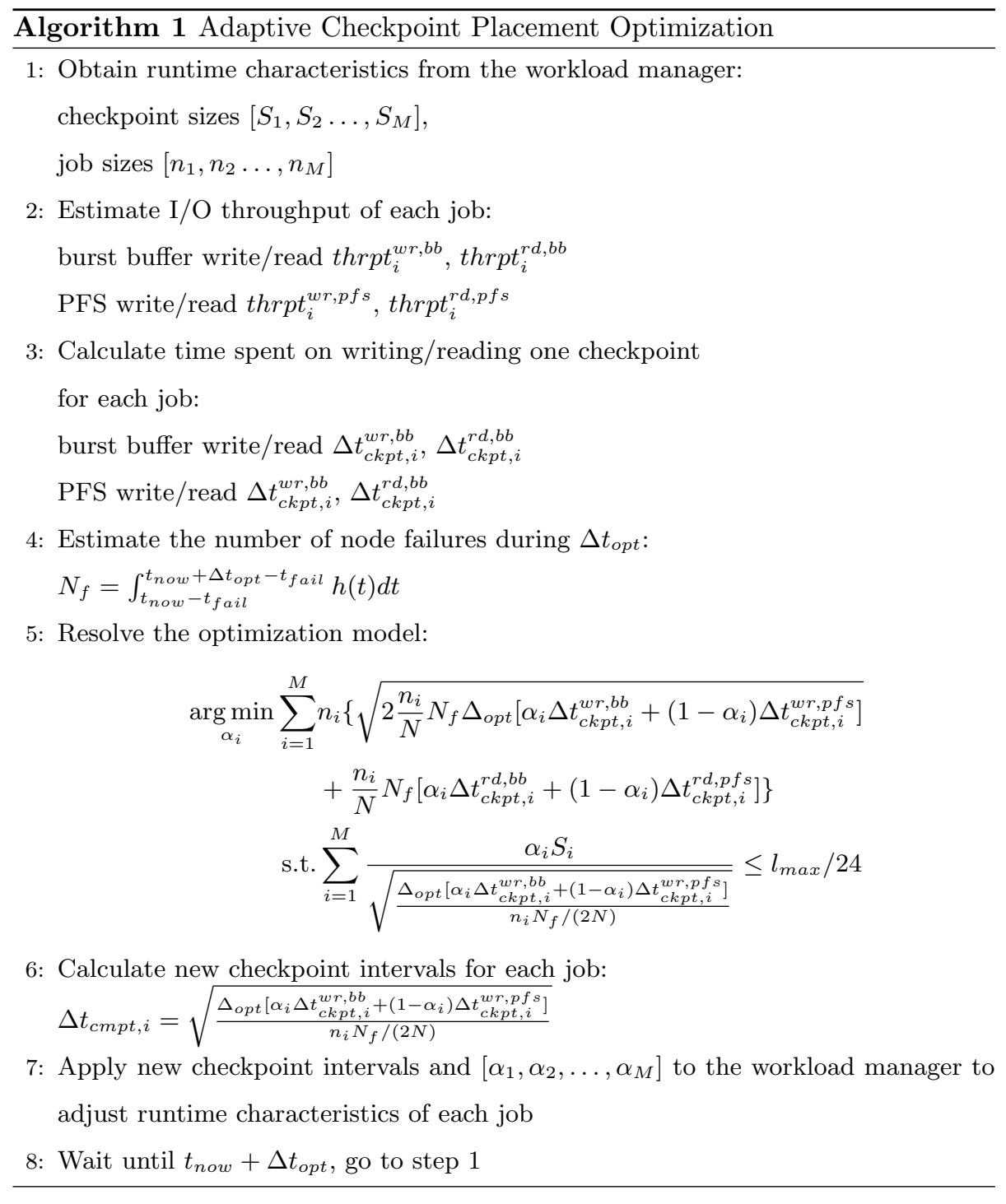

Furthermore, the constraint on burst buffer write limit can be denoted as follows:

$$
\sum_{i=1}^{M} \frac{\alpha_{i} S_{i}}{\sqrt{\frac{\Delta_{o p t}\left[\alpha_{i} \Delta t_{c k p t, i}^{w r, b b}+\left(1-\alpha_{i}\right) \Delta t_{c k p t, i}^{w r, p f s}\right]}{n_{i} N_{f} /(2 N)}}} \leq l_{\max } / 24
$$

As illustrated in Algorithm 1, our algorithm will be called by the workload manager to optimize the checkpoint placement at the beginning of every $\Delta t_{\text {opt }}$. Specifically, our algorithm first obtains the runtime characteristics of each run- 
ning job from the workload manager, including the checkpoint size and job size. Then based on the job size, it estimates the I/O throughput each job can achieve when writing the checkpoints to or reading the checkpoints from the storage. For example, the burst buffer layers in HPC systems are usually built upon SSD devices locally attached to each compute node. Therefore, the aggregate I/O throughput the job can achieve is roughly estimated as the product of the job size and each SSD's I/O bandwidth. For the parallel file system, such as Spider II, when the job size is below some threshold, the aggregate I/O throughput is also proportional to the job size, but once the job size is larger than such threshold, the I/O bandwidth of the parallel file system will be saturated [38]. After estimating the I/O throughput, our algorithm calculates the time spent on writing one checkpoint for each job.

The most challenging part in our algorithm is estimating the number of node failures rate in the HPC system. Most large-scale HPC systems maintain failure logs during their operation $[10,39,40]$. By analyzing the failure logs, we can obtain the failure characteristics of compute nodes in the system. For example, as we mentioned in previous paragraphs, the analysis of failure logs demonstrates a Weibull distribution with decreasing failure rate fits the time between failures of Titan supercomputer the best. By estimating the parameters of such Weibull distribution, we can also obtain the hazard rate function, $h(t)$.

Finally, with all the runtime characteristics that have been obtained, estimated or calculated, our algorithm resolves the optimization model to get new percentage of checkpoints that should be written to the burst buffer as well as the new checkpoint interval for each job. These optimization results will be applied to all jobs through the workload manager to guide the checkpoint placement in the coming period.

\section{Evaluation}

In this section, we evaluate our adaptive checkpoint placement algorithm through event-based simulation and the failure traces collected from Titan su- 
percomputer are used to drive the simulation.

\subsection{Evaluation Setup}

Our simulation parameter settings are based on system configuration settings of deployed leadership computing facilities and runtime characteristics obtained from real scientific application jobs.

\subsubsection{System features of leadership computing facilities}

- Compute platform: Our models and algorithms are primarily evaluated based on the architecture and characteristics of Titan, the second fastest supercomputer in the world, which is deployed and managed by OLCF. Titan is composed of 18,688 compute nodes and has more than $700 \mathrm{~TB}$ memory capacity. According to the failure data collected by OLCF, the mean time between failure (MTBF) of the entire Titan supercomputer is about 11 hours and the time between node failures satisfies a Weibull distribution (See TABLE 2). In our evaluation, we use the Titan failure data to drive the compute node failures in our simulation.

- Burst buffer: Since Titan does not have a burst buffer layer per se, we apply system characteristics of Gordon supercomputer [41], one of the first supercomputers utilizing SSDs, to our evaluation.

- SSD model: The solid state drives used to equip Gordon supercomputer are Intel 710 (300GB) SSDs, which are relatively expensive compared to other manufactures' products. For example, each Samsung 850 Pro (256GB) SSD [7] costs $\$ 120$, only one forth of the list price of the Intel 710 (300GB) SSD. Since Titan has much more compute nodes than Gordon, we choose Samsung 850 Pro (256GB) SSDs to build a more cost-effective burst buffer for Titan in our evaluation, even though some expensive SSDs might provide better performance and longer endurance. 
- Capacity: The burst buffer layer of Gordon consists of $64 \mathrm{I} / \mathrm{O}$ nodes. Each of these I/O nodes contains 16 SSDs, and serves 16 compute nodes. In our evaluation, we also assume that averagely each compute node of Titan can be served by one SSD, regardless of whether such SSD is integrated into the I/O node or locally attached to the compute node. Therefore, the total capacity of the burst buffer in our evaluation is about $256 \times 18688=4.6 \mathrm{~PB}$.

- Bandwidth: As the 64 I/O nodes (the burst buffer layer) of Gordon system can provide $320 \mathrm{~GB} / \mathrm{s}$ aggregate write bandwidth, each SSD can roughly provide $320 \mathrm{MB} / \mathrm{s}$ write bandwidth. Let us assume each compute node of Titan supercomputer can also enjoy a similar write bandwidth. Since Titan has 18,688 compute nodes in total, the aggregate write bandwidth of the burst buffer will be around $320 \times 18688 / 1024=5,840 \mathrm{~GB} / \mathrm{s}$.

- Write limit: If each compute node is served by a dedicated SSD, then the total number of SSDs in Titan's burst buffer is 18,688. Since each Samsung 850 Pro SSD has a warranty for maximum 150TB write, if the burst buffer is designed to be in operation for at least 5 years, the maximum amount of data can be written to the burst buffer per day is around $(150 \times 18688) /(5 \times 365)=1,536 \mathrm{~TB}$. If the write amplification factor is about 1.3, then the actual write limit should be $1536 / 1.3=1,100 \mathrm{~TB}$ per day. In all evaluation cases, we set the daily write limit of the burst buffer as $1,000 \mathrm{~TB}$.

- Parallel file system: Spider II file system is a Lustre-based parallel file system used by Titan supercomputer. It consists of 20,106 hard disk drives and provides $32 \mathrm{~PB}$ capacity (after RAID). The aggregate I/O bandwidth of Spider II file system depends on the number of clients issuing the I/O operations concurrently. According to measurement results provided by OLCF [38], the aggregate write bandwidth of Spider II can linearly increase up to $300 \mathrm{~GB} / \mathrm{s}$ when the number of clients is less than 6,000 . Once 
more than 6,000 clients are writing data to Spider II concurrently, the I/O bandwidth gets saturated.

\subsubsection{Runtime characteristics of scientific applications}

- Checkpoint size: Different scientific applications usually write checkpoints of different sizes and the difference could be huge. For example, CHIMERA and VULCAN are two scientific applications running on Titan supercomputer. Each checkpoint of CHIMERA is 160TB while that of VULCAN is only $0.83 \mathrm{~GB}$.

- Job size: Job size means the number of compute nodes each computation job occupies. Job size not only determines the aggregate I/O bandwidth of writing and reading checkpoints, but also effects the failure rate of each running scientific application. In our simulation, we vary the job size from 256 to 9216 to cover the wide range of job characteristics in real HPC environment.

- Computation time: Computation time of each scientific application is the time required to finish all computation task, not including that spent on writing checkpoints or recovering from failures. It depends on the complexity of the job and number of compute nodes used by the job. In our simulation, the computation time of simulated scientific applications varies from 120 to 720 hours.

TABLE 3 lists all runtime characteristics of several common scientific applications running on Titan supercomputer, including the size of each checkpoint generated by each scientific application, the number of compute nodes occupied by each application, and the total computation time required to complete each application. We apply these characteristics to our evaluation and simulate the computation/checkpointing activities of these scientific applications when they are running concurrently on Titan supercomputer. 


\begin{tabular}{|l|l|l|l|l|}
\hline Domain & Application & Checkpoint size & Job size & Computation time \\
\hline Astrophysics & CHIMERA & $160 \mathrm{~TB}$ & 9216 & 360 Hours \\
\hline Astrophysics & VULCAN & $0.83 \mathrm{~GB}$ & 256 & 720 Hours \\
\hline Climate & POP & $26 \mathrm{~GB}$ & 512 & 480 Hours \\
\hline Combustion & S3D & $5 \mathrm{~TB}$ & 2048 & 240 Hours \\
\hline Fusion & GTC & $20 \mathrm{~TB}$ & 6144 & 120 Hours \\
\hline Fusion & GYRO & $50 \mathrm{~GB}$ & 512 & 120 Hours \\
\hline
\end{tabular}

Table 3: Runtime characteristics of common scientific applications running on Titan supercomputer

\subsubsection{Evaluated Models and Algorithms}

In order to demonstrate the effectiveness of our adaptive checkpoint placement algorithm, we also evaluate several other models and algorithms for comparison. All the models and algorithms we evaluate are listed in TABLE 4 and the detailed description of these models are given as follows. When model SUBNP is used, all the checkpoint data is written to the burst buffer without limit while no checkpoint is written to the PFS directly, and the checkpoint interval is determined through one-time calculation using formula (2) (see section 3.1). When model AUBNP is used, all the checkpoint data is written to the burst buffer without limit while no checkpoint is written to the PFS directly, and the checkpoint interval is determined through adaptive algorithm named "lazy checkpoint" (see section 3.2). When model SLBNP is used, limited checkpoint data is written to the burst buffer while no checkpoint is written to the PFS directly, and the checkpoint interval is determined through the optimization model proposed in [14] (see section 3.3). SLBUP and ALBUP are two models proposed in this paper, which all limit the checkpoint data written to the burst buffer while leverage the PFS to keep the checkpoint frequency from decreasing too much (see section 4 and 5 for more details).

In order to have a fair comparison, the system features of the leadership computing facility and the runtime characteristics of those scientific computation jobs are kept the same when evaluating each of these models and algorithms. 


\begin{tabular}{|c|c|}
\hline Abbreviation & Description of the model or algorithm \\
\hline $\begin{array}{l}\text { Static-Unlim-BB-No-PFS } \\
(\text { SUBNP })\end{array}$ & $\begin{array}{l}\text { Static optimal checkpoint interval given by formula (2) } \\
\text { is used. All checkpoints are written to the burst buffer } \\
\text { without limit. No checkpoint is written to the PFS directly. }\end{array}$ \\
\hline $\begin{array}{l}\text { Adapt-Unlim-BB-No-PFS } \\
(\text { AUBNP) }\end{array}$ & $\begin{array}{l}\text { Adaptive checkpoint model proposed by }[10] \text { is used. } \\
\text { All checkpoints are written to the burst buffer without } \\
\text { limit. No checkpoint is written to the PFS directly. }\end{array}$ \\
\hline $\begin{array}{l}\text { Static-Lim-BB-No-PFS } \\
(\text { SLBNP })\end{array}$ & $\begin{array}{l}\text { Static checkpoint interval from the model proposed } \\
\text { by }[14] \text { is used. Limited checkpoints are written to the } \\
\text { burst buffer. No checkpoint is written to the PFS directly. }\end{array}$ \\
\hline $\begin{array}{l}\text { Static-Lim-BB-Unlim-PFS } \\
\text { (SLBUP) }\end{array}$ & $\begin{array}{l}\text { Our static checkpoint placement model with guaranteed } \\
\text { burst buffer endurance (see formula (13)) is used. } \\
\text { Limited checkpoints are written to the burst buffer. } \\
\text { Unlimited checkpoints can be written to the PFS directly. }\end{array}$ \\
\hline $\begin{array}{l}\text { Adapt-Lim-BB-Unlim-PFS } \\
\text { (ALBUP) }\end{array}$ & $\begin{array}{l}\text { Our adaptive checkpoint placement algorithm is used. } \\
\text { Limited checkpoints are written to the burst buffer. } \\
\text { Unlimited checkpoints can be written to the PFS directly. }\end{array}$ \\
\hline
\end{tabular}

Table 4: Evaluated models and algorithms

\subsection{Evaluation Results}

\subsubsection{I/O workloads the burst buffer burdens with}

We first evaluate the average I/O workloads the burst buffer burdens with when different checkpoint placement models or algorithms are used by the scientific computation jobs. Specifically, in the evaluation, we simulate the checkpoint/restart activities of all jobs listed in TABLE 3 during 100-hour operation of Titan and calculate the average amount of checkpoint data written to and read from the burst buffer. During this 100-hour operation, except the node failure rate, all other runtime characteristics, such as the number of jobs, the size of each job, etc., do not vary with time. The evaluation result is shown in Figure 4.

Model SUBNP means all checkpoints from all jobs running on Titan are written to the burst buffer directly without limit and formula (2) is adopted to determine the optimal checkpoint interval for each job, as shown in Figure 


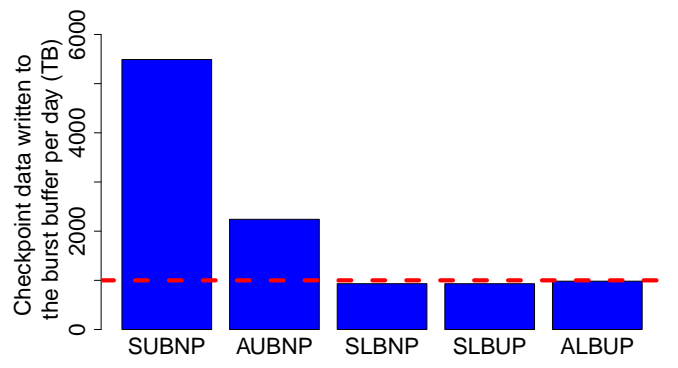

(a) Write workloads (the dash line represents the daily write limit of the burst buffer for keeping a 5 -year operation lifetime)

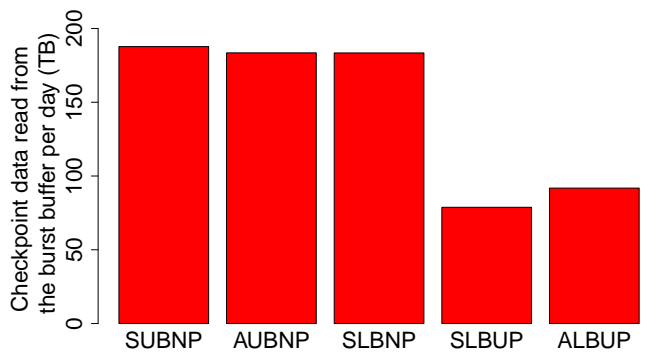

(b) Read workloads

Figure 4: Average amount of checkpoint data written to and read from the burst buffer per day when different checkpoint placement models or algorithms are used

4(a), the amount of checkpoint data written to the burst buffer per day could be more than 5,500TB. If the burst buffer is built with Samsung 850 Pro SSDs, under such intensive write workload, the lifetime of the burst buffer might be only $(150 \times 18688) /(5500 \times 365)=1.4$ years. Even if model AUBNP is used, which can dynamically adjust the checkpoint interval according to the changing node failure rate to reduce the write workloads, as long as all checkpoints are directly absorbed by the burst buffer, the data written to the burst buffer per day is still about $2,200 \mathrm{~TB}$, much more than that is allowed if the burst buffer is expected to serve more than 5 years. Therefore, in order to prolong the lifetime of the burst buffer, write workloads issued to the burst buffer must be limited. For example, since the write limit of the burst buffer has been taken into consideration, when model SLBNP, SLBUP and algorithm ALBUP 
are used, the daily write workloads issued to the burst buffer are all less than 1,000TB (the dash line in Figure 4(a)) and the lifetime of the burst buffer can be extended to more than 5 years.

In Figure 4(b), we also illustrate the average amount of checkpoint data read from the burst buffer per day when different checkpoint placement models are used. Apparently, since model SUBNP, AUBNP and SLBNP allow all checkpoints to be written to the burst buffer directly without limit, the jobs always restart from failures using checkpoints stored on the burst buffer. That is why the average amount of data read from the burst buffer per day when using these three models are similar. For model SLBUP and algorithm ALBUP, the read workloads issued to the burst buffer are much less, as some of the checkpoints have been written to the parallel file system instead of the burst buffer. Moreover, using algorithm ALBUP generates 16.5\% more read workloads than model SLBUP. This is because algorithm ALBUP dynamically adjusts the percentage of checkpoints written to the burst buffer based on the changing node failure rate and increases the likelihood of restarting the job using checkpoints stored on the burst buffer.

\subsubsection{Computation efficiency of the scientific applications and entire HPC sys-} tem

Next we evaluate the computation efficiency of each scientific application job as well as that of the entire HPC system when different checkpoint placement models are applied to.

The computation efficiency of the $i$-th scientific application is defined as follows:

$$
i \text {-th job's computation efficiency }=\frac{T_{c m p t, i}}{T_{t o t a l, i}} \times 100 \%,
$$

where $T_{\text {total }, i}$ is the total execution time and $T_{c m p t, i}$ is the total time spent on computation. As shown in Figure 5, if model SUBNP is used, each scientific application job can achieve the best computation efficiency. Compared to model SUBNP, using model AUBNP results in a slightly decrease in the computation efficiency as the dynamic adjustment of checkpoint interval might potentially in- 


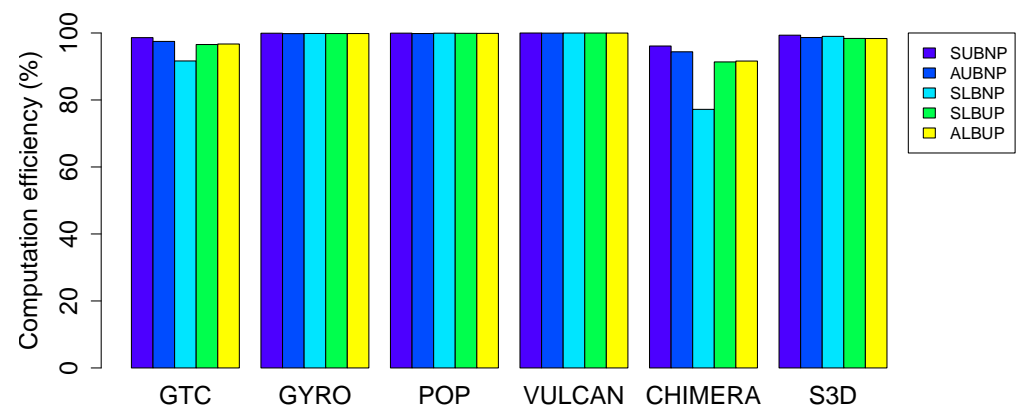

Figure 5: Average computation efficiency of each scientific application job when different checkpoint placement models or algorithms are used

crease the wasted computation time. However, both model SUBNP and AUBNP cannot satisfy the burst buffer endurance requirement. If the write limits of the burst buffer is set as 1,000TB per day, using model SLBNP reduces the computation efficiency of jobs that generate large checkpoints (such as CHIMERA and GTC) significantly. This is because such model intends to increase the checkpoint intervals of those jobs to reduce the amount of data written to the burst buffer, which inevitably increases the wasted time due to potential failures of compute nodes. On the other hand, with the same write limits, if our SLBUP model or ALBUP algorithm is used, computation efficiency of each job at most decreases by $5 \%$ compared to model SUBNP. This is because these two models utilize both burst buffer and the underlying parallel file system collaboratively to store checkpoint data and can keep the original optimal checkpoint interval to some extent without exceeding the write limit of the burst buffer.

The computation efficiency of the entire HPC system is defined as follows:

$$
\text { system computation efficiency }=\frac{\sum_{i=1}^{M} n_{i} T_{c m p t, i}}{\sum_{i=1}^{M} n_{i} T_{\text {total }, i}} \times 100 \%,
$$

where $M$ is the number of jobs running on the HPC system and $n_{i}$ is the size of the $i$-th job. Therefore, after obtaining the average total execution time and computation time of each scientific application job from the simulation, we can calculate the system computation efficiency when different checkpoint placement models or algorithms are used. The calculation results are listed in TABLE 5. 


\begin{tabular}{|l|l|}
\hline Model/algorithm & System efficiency (\%) \\
\hline SUBNP & 97.55 \\
\hline AUBNP & 96.22 \\
\hline SLBNP & 84.57 \\
\hline SLBUP & 94.40 \\
\hline ALBUP & 94.58 \\
\hline
\end{tabular}

Table 5: System computation efficiency when different checkpoint placement models or algorithms are used

The calculation results demonstrate that, compared to model SUBNP, the system efficiency decreases by $13 \%$ when model SLBNP is used, while using our SLBUP model or ALBUP algorithm, the system efficiency at most decreases by $3 \%$.

\subsubsection{Comparison of static vs. adaptive checkpoint placement}

Since some runtime characteristics, such as the failure rate and job sizes, might vary with time during the execution of the scientific applications, the checkpoint placement optimization needs to adapt to the changing runtime characteristics to achieve the most efficient utilization of the burst buffer. Next, we present the comparison between the static and the adaptive checkpoint placement when the runtime characteristics are not constant during the operation of the HPC system.

First, let us study how static and adaptive checkpoint placement perform when the node failure rate is time-dependent. In order to obtain a stable and accurate estimation on different models' performance, we need to run the simulation many times and use the average of all simulation runs' results as our evaluation results. Therefore, besides the actual failure traces we collected from Titan, we also use the Weibull distribution that fits the Titan's failure logs best (see TABLE 2 and Figure 2) to generate synthetic failure traces to drive each run of the simulation. The simulation results produced by using our synthetic failure traces approximate to that using the actual failure traces well. 


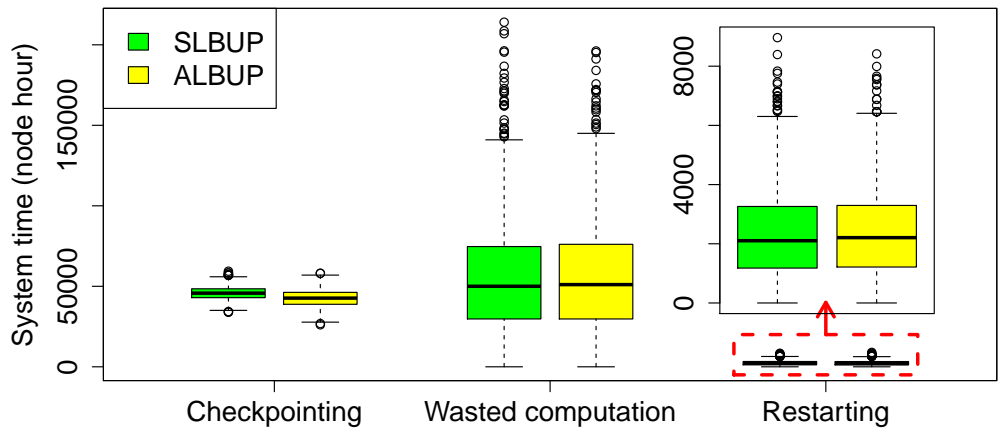

Figure 6: Box plot of system time spent on writing checkpoints, wasted computation due to failures and job restarting, when the node failure rate is time-dependent (1,000 simulation runs for each model)

As shown in TABLE 5, though not much, our adaptive checkpoint placement algorithm ALBUP does achieve higher system computation efficiency than the static model SLBUP. The reason that algorithm ALBUP performs better than model SLBUP when the failure rate is time-dependent can be illustrated using Figure 6. In Figure 6, we adopt box plot to visualize the distribution of different runtime overhead observed in 1,000 simulation runs of each model, including system time spent on writing checkpoints, wasted computation due to failures and job restarting. From this figure, we can observe that: 1) Algorithm ALBUP can reduce the system time spent on checkpointing by almost 8\% (3,400 node hour). This is because ALBUP can dynamically adjust the checkpoint intervals for each running job based on the time-dependent node failure rate and reduce the number of checkpoints written to the storage. 2) Though the number of checkpoints reduces when ALBUP algorithm is used, the time spent on wasted computation and restarting the failed jobs have no obvious change compared to model SLBUP. This means the checkpointing overhead is reduced without increasing other overhead. 3) Actually, when we calculate the expectations of these two distributions, it shows that the average wasted computation time is reduced by 175 node hour while the average time spent on restarting is decreased by 72 node hour if ALBUP is used, which indicates that our ALBUP algorithm 


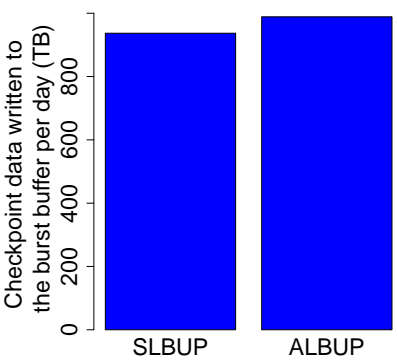

(a) Write workloads

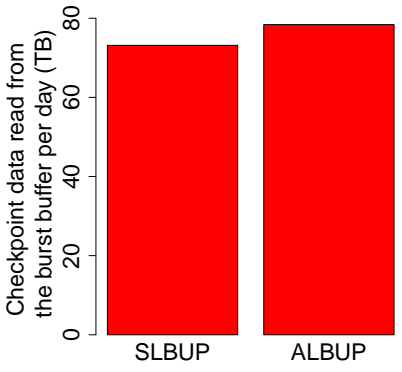

(b) Read workloads

Figure 7: Average amount of checkpoint data written to and read from the burst buffer per day when job sizes are varying over time

can perform checkpoint placement more efficiently compared to our SLBUP model.

Second, we evaluate how static and adaptive checkpoint placement perform when the job sizes are varying over time. In our evaluation, we assume that once a job fails, the chance it will lose some nodes after restarting is $70 \%$ and the percentage of nodes it will lose is a random variable whose value is selected between 0 and 10\%. Then we run the simulation for model SLBUP and algorithm ALBUP respectively and the results are shown in Figure 7.

As shown in Figure 7(a), when the job sizes are varying over time, the total amount of checkpoint data written to the burst buffer is only 930TB per day if the static checkpoint placement is used, $7 \%$ less than the 1,000TB per day write limit, which means the burst buffer is not fully utilized. However, if the adaptive checkpoint placement is used, the daily write workloads to the burst buffer is still around 1,000TB per day. From Figure 7(b), we can also observe similar phenomenon on the total amount of checkpoint data read from the burst buffer. This is because the ALBUP algorithm can dynamically adjust the percentage of checkpoints written to the burst buffer based on the changing job sizes so that the burst buffer can always be fully utilized. Besides, we also calculate the average system computation efficiency when the job sizes are varying over time. The calculation results indicate that using model SLBUP the system efficiency 
is $94.67 \%$, while using the ALBUP algorithm the system can achieve $94.75 \%$ efficiency.

\subsubsection{Effect of optimization period on adaptive checkpoint placement}

Since our ALBUP algorithm is designed to be triggered periodically to optimize the checkpoint placement, the length of the time period between two consecutive calls of ALBUP might have non-negligible impact on the model's performance. Therefore, we also evaluate our ALBUP algorithm by varying the period of triggering it during system operation.

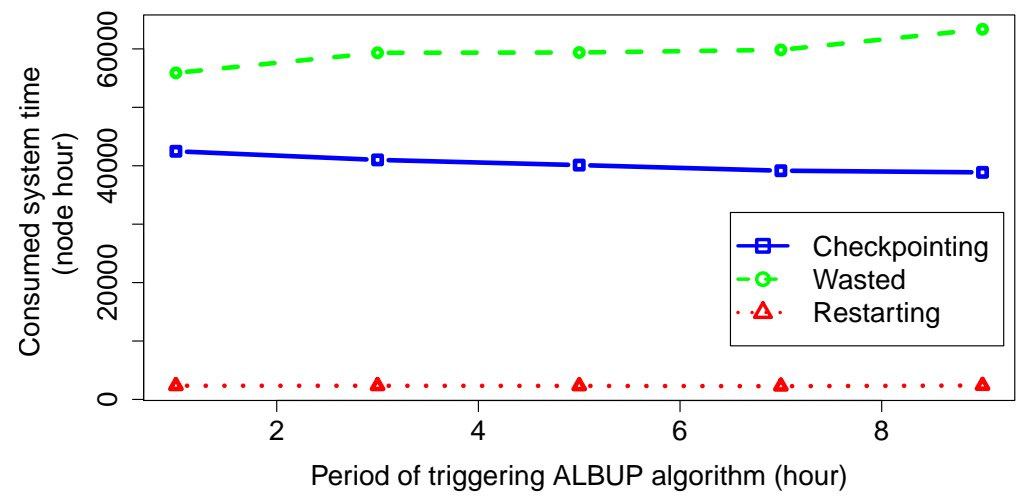

Figure 8: Performance of the ALBUP algorithm when varying the period between optimizations

As shown in Figure 8, when we increase the period of calling the ALBUP algorithm from 1 hour to 9 hours, we obtain the following results: 1) The average system time spent on checkpointing decreases. Specifically, the increase of the optimization period results in underestimate on the number of node failures during each period, which makes the algorithm incorrectly extend the checkpoint intervals. Since less checkpoints are written, the time consumed by the checkpointing operation decreases. 2) The average wasted computation time increases significantly. This is also caused by the increase of the checkpoint interval, as more failed jobs have to restart using checkpoints that were not recently written. 3) The system time spent on restarting the failed jobs has no obvious variation. 4) The system computation efficiency does not decrease 
significantly until the optimization period is longer than 9 hours.

\section{Conclusions and Future Work}

The storage hierarchy in large-scale HPC system has been deepened with the introduction of the SSD-based burst buffer layer. SSD endurance in itself is already a critical concern for enterprise and data center computing environment. The problem is amplified even more when we consider that its major use case in HPC storage hierarchy is to handle write-intensive I/O workloads such as checkpointing. If no safeguards are in place, workloads at extreme scale and intensity could potentially render SSD devices inoperable at much faster pace than anticipated, which not only drive up the operational cost, but also negatively impact the overall system stability.

In this paper, we propose a new checkpoint placement optimization model which collaboratively utilizes both burst buffer and parallel file system layers to store the checkpoints. Our design goal is to maximize the computation efficiency while guaranteeing the SSD endurance requirements. We also present an adaptive algorithm which can adjust the checkpoint placement based on the dynamically changing runtime HPC system characteristics, while continuously optimizing the burst buffer utilization. Evaluation results show that both our checkpoint placement model and adaptive algorithm can guarantee the burst buffer endurance without degrading more than $3 \%$ system performance. The evaluation results also show that our adaptive algorithm is able to satisfy the endurance requirements, and also fully utilizes the burst buffer to maximize the computation efficiency even the runtime characteristics are changing over time.

In our future work, we will analyze the I/O access patterns of different HPC applications and study how these I/O access patterns affect the SSD lifetime. Besides, we will also carry on more evaluation using different models of SSD devices to provide the storage system designers more insight into the endurance variation among devices and help them make better design choices. 


\section{Acknowledgement}

This research used resources of the Oak Ridge Leadership Computing Facility, which is a DOE Office of Science User Facility supported under Contract DE-AC05-00OR22725.

\section{References}

[1] N. Liu, J. Cope, P. H. Carns, C. D. Carothers, R. B. Ross, G. Grider, A. Crume, C. Maltzahn, On the Role of Burst Buffers in Leadership-Class Storage Systems, in: 28th Symposium on Mass Storage Systems and Technologies, MSST '12, 2012, pp. 1-11.

[2] K. Sato, N. Maruyama, K. Mohror, A. Moody, T. Gamblin, B. R. de Supinski, S. Matsuoka, Design and Modeling of a Non-Blocking Checkpointing System, in: Proceedings of the International Conference on High Performance Computing, Networking, Storage and Analysis, SC '12, 2012, pp. 19:1-19:10.

[3] J. Bent, S. Faibish, J. Ahrens, G. Grider, J. Patchett, P. Tzelnic, J. Woodring, Jitter-Free Co-Processing on a Prototype Exascale Storage Stack, in: 28th Symposium on Mass Storage Systems and Technologies, MSST '12, 2012, pp. 1-5.

[4] W. Xu, Y. Lu, Q. Li, E. Zhou, Z. Song, Y. Dong, W. Zhang, D. Wei, X. Zhang, H. Chen, J. Xing, Y. Yuan, Hybrid Hierarchy Storage System in MilkyWay-2 Supercomputer, Frontiers of Computer Science 8 (3) (2014) 367-377.

[5] K. Sato, K. Mohror, A. Moody, T. Gamblin, B. R. d. Supinski, N. Maruyama, S. Matsuoka, A User-Level InfiniBand-Based File System and Checkpoint Strategy for Burst Buffers, in: 14th IEEE/ACM International Symposium on Cluster, Cloud and Grid Computing, CCGrid '14, 2014, pp. 21-30. 
[6] X. Hu, E. Eleftheriou, R. Haas, I. Iliadis, R. Pletka, Write Amplification Analysis in Flash-Based Solid State Drives, in: Proceedings of SYSTOR 2009: The Israeli Experimental Systems Conference, SYSTOR '09, 2009, pp. 10:1-10:9.

[7] Samsung, Samsung SSD 850 PRO Series Datasheet (2015).

URL http://www.samsung.com/global/business/semiconductor/ minisite/SSD/downloads/document/Samsung_SSD_850_PRO_Data_ Sheet_rev_2_0.pdf

[8] OLCF, ORNL Debuts Titan Supercomputer (2012).

URL https://www.olcf.ornl.gov/wp-content/themes/olcf/titan/ Titan_Debuts.pdf

[9] UCSF, UCSF CHIMERA: an Extensible Molecular Modeling System (2015).

URL http://www.rbvi.ucsf .edu/chimera/

[10] D. Tiwari, S. Gupta, S. S. Vazhkudai, Lazy Checkpointing: Exploiting Temporal Locality in Failures to Mitigate Checkpointing Overheads on Extreme-Scale Systems, in: 44th Annual IEEE/IFIP International Conference on Dependable Systems and Networks, DSN '14, 2014, pp. 25-36.

[11] Q. Yang, J. Ren, I-CASH: Intelligently Coupled Array of SSD and HDD, in: 17th International Conference on High-Performance Computer Architecture, HPCA '15, 2011, pp. 278-289.

[12] S. Lee, T. Kim, K. Kim, J. Kim, Lifetime Management of Flash-Based SSDs Using Recovery-Aware Dynamic Throttling, in: Proceedings of the 10th USENIX Conference on File and Storage Technologies, FAST '12, 2012, pp. 26-26.

[13] J. Kaiser, F. Margaglia, A. Brinkmann, Extending SSD Lifetime in Database Applications with Page Overwrites, in: Proceedings of the 6th 
International Systems and Storage Conference, SYSTOR '13, 2013, pp. 11:1-11:12.

[14] A. Fang, A. A. Chien, How Much SSD Is Useful for Resilience in Supercomputers, in: Proceedings of the 5th Workshop on Fault Tolerance for HPC at eXtreme Scale, FTXS '15, 2015, pp. 47-54.

[15] A. Moody, G. Bronevetsky, K. Mohror, B. R. d. Supinski, Design, Modeling, and Evaluation of a Scalable Multi-Level Checkpointing System, in: Proceedings of the 2010 ACM/IEEE International Conference for High Performance Computing, Networking, Storage and Analysis, SC '10, 2010, pp. $1-11$.

[16] T. Wang, S. Oral, Y. Wang, B. W. Settlemyer, S. Atchley, W. Yu, BurstMem: a High-Performance Burst Buffer System for Scientific Applications, in: International Conference on Big Data, 2014, pp. 71-79.

[17] S. Park, K. Shen, A Performance Evaluation of Scientific I/O Workloads on Flash-Based SSDs, in: Proceedings of the International Conference on Cluster Computing, CLUSTER '09, 2009, pp. 1-5.

[18] N. Agrawal, V. Prabhakaran, T. Wobber, J. D. Davis, M. Manasse, R. Panigrahy, Design Tradeoffs for SSD Performance, in: USENIX 2008 Annual Technical Conference, ATC '08, 2008, pp. 57-70.

[19] F. Chen, T. Luo, X. Zhang, CAFTL: A Content-aware Flash Translation Layer Enhancing the Lifespan of Flash Memory Based Solid State Drives, in: Proceedings of the 9th USENIX Conference on File and Stroage Technologies, FAST '11, 2011, pp. 6-6.

[20] G. Wu, X. He, Delta-FTL: Improving SSD Lifetime via Exploiting Content Locality, in: Proceedings of the 7th ACM European Conference on Computer Systems, EuroSys '12, 2012, pp. 253-266. 
[21] P.-L. Wu, Y.-H. Chang, T.-W. Kuo, A File-System-Aware FTL Design for Flash-Memory Storage Systems, in: Proceedings of the Conference on Design, Automation and Test in Europe, DATE '09, 2009, pp. 393-398.

[22] Y. Lu, J. Shu, W. Zheng, Extending the Lifetime of Flash-Based Storage Through Reducing Write Amplification from File Systems, in: Proceedings of the 11th USENIX Conference on File and Storage Technologies, FAST '13, 2013, pp. 257-270.

[23] G. Soundararajan, V. Prabhakaran, M. Balakrishnan, T. Wobber, Extending SSD Lifetimes with Disk-based Write Caches, in: Proceedings of the 8th USENIX Conference on File and Storage Technologies, FAST '10, 2010, pp. 8-8.

[24] F. Chen, D. A. Koufaty, X. Zhang, Hystor: Making the Best Use of Solid State Drives in High Performance Storage Systems, in: Proceedings of the International Conference on Supercomputing, ICS '11, ACM, New York, NY, USA, 2011, pp. 22-32.

[25] L. Wan, Z. Lu, Q. Cao, F. Wang, S. Oral, B. Settlemyer, SSD-Optimized Workload Placement with Adaptive Learning and Classification in HPC Environments, in: 30th Symposium on Mass Storage Systems and Technologies, MSST '14, 2014, pp. 1-6.

[26] J. W. Young, A First Order Approximation to the Optimum Checkpoint Interval, Communications of the ACM 17 (9) (1974) 530-531.

[27] N. H. Vaidya, Impact of Checkpoint Latency on Overhead Ratio of a Checkpointing Scheme, IEEE Transactions on Computers 46 (8) (1997) 942-947.

[28] J. T. Daly, A Higher Order Estimate of the Optimum Checkpoint Interval for Restart Dumps, Future Generation Computer Systems 22 (3) (2006) 303-312.

[29] Y. Liu, R. Nassar, C. Leangsuksun, N. Naksinehaboon, M. Paun, S. Scott, A Reliability-Aware Approach for An Optimal Checkpoint/Restart Model 
in HPC Environments, in: IEEE International Conference on Cluster Computing, CLUSTER '07, 2007, pp. 452-457.

[30] M. Bougeret, H. Casanova, M. Rabie, Y. Robert, F. Vivien, Checkpointing Strategies for Parallel Jobs, in: Proceedings of 2011 International Conference for High Performance Computing, Networking, Storage and Analysis, SC '11, 2011, pp. 33:1-33:11.

[31] N. El-Sayed, B. Schroeder, Checkpoint/Restart in Practice: When Simple is Better, in: IEEE International Conference on Cluster Computing, CLUSTER '14, 2014, pp. 84-92.

[32] A. Wächter, L. T. Biegler, On the Implementation of an Interior-point Filter Line-Search Algorithm for Large-Scale Nonlinear Programming, Mathematical Programming 106 (1) (2006) 25-57.

[33] B. Schroeder, G. A. Gibson, A Large-Scale Study of Failures in HighPerformance Computing Systems, in: Proceedings of the International Conference on Dependable Systems and Networks, DSN '06, 2006, pp. 249-258.

[34] N. El-Sayed, B. Schroeder, To Checkpoint or Not To Checkpoint: Understanding Energy-Performance-I/O Tradeoffs in HPC Checkpointing, in: Proceedings of the International Conference on Cluster Computing, CLUSTER '14, 2014, pp. 93-102.

[35] B. Schroeder, G. Gibson, The Computer Failure Data Repository (CFDR): Collecting, Sharing and Analyzing Failure Data, in: Proceedings of the International Conference for High Performance Computing, Networking, Storage and Analysis, SC '06, 2006.

[36] SchedMD, Slurm Workload Manager (2015).

URL http://slurm.schedmd.com/

[37] LBNL, Berkeley Lab Checkpoint/Restart (BLCR) for LINUX (2015).

URL http://crd.lbl.gov/departments/computer-science/CLaSS/ research/BLCR/ 
[38] S. Oral, J. Simmons, J. Hill, D. Leverman, F. Wang, M. Ezell, R. Miller, D. Fuller, R. Gunasekaran, Y. Kim, S. Gupta, D. Tiwari, S. S. Vazhkudai, J. H. Rogers, D. Dillow, G. M. Shipman, A. S. Bland, Best Practices and Lessons Learned from Deploying and Operating Large-Scale Data-Centric Parallel File Systems, in: Proceedings of the International Conference for High Performance Computing, Networking, Storage and Analysis, SC '14, 2014, pp. 217-228.

[39] L. Wan, F. Wang, S. Oral, D. Tiwari, S. S. Vazhkudai, Q. Cao, A Practical Approach to Reconciling Availability, Performance, and Capacity in Provisioning Extreme-Scale Storage Systems, in: Proceedings of the International Conference for High Performance Computing, Networking, Storage and Analysis, SC '15, 2015, pp. 75:1-75:12.

[40] L. Wan, F. Wang, S. Oral, S. S. Vazhkudai, Q. Cao, A Report on Simulation-Driven Reliability and Failure Analysis of Large-Scale Storage Systems, Tech. Rep. ORNL/TM-2014/421, Oak Ridge National Laboratory (December 2014).

[41] SDSC, Gordon Supercomputer (2015).

URL http://www.sdsc.edu/support/user_guides/gordon.html 